\title{
EFECTO DE UN PLAN DE ENTRENAMIENTO FÍSICO EN EL CONSUMO MÁXIMO DE OXÍGENO (VO2MÁX) Y EL PESO EN PACIENTES HIPERTENSOS ADULTOS MAYORES ADSCRITOS A LA IPS CAJASAN (BUCARAMANGA)
}

\author{
PHYSICAL TRAINING PROGRAM EFFECT IN THE UPTAKE OF MAXIMUM OXYGEN (VO2MÁx) AND \\ ELDERLY HYPERTENSIVE PATIENTS' WEIGHT, ASSIGNED TO THE IPS CAJASAN OF BUCARAMANGA
}

\section{Resumen}

\section{Abstract}

\begin{abstract}
El ejercicio físico adquiere cada vez más importancia en el tratamiento de los pacientes con hipertensión arterial, sobrepeso y obesidad. Desde hace más de cinco décadas se sabe que el ejercicio físico tiene efectos favorables en el tratamiento de la hipertensión arterial y otros factores de riesgo, es coadyuvante en el tratamiento de las enfermedades cardiovasculares y favorece el consumo máximo de oxígeno (VO2máx). El objetivo de este trabajo fue determinar el efecto de un plan de entrenamiento físico sobre el V02máx y el peso en adultos mayores hipertensos adscritos a la IPS Cajasan Bucaramanga. Es una investigación cuasiexperimental, se tomaron cuarenta pacientes entre 60 y 75 años, en dos grupos iguales, uno con entrenamiento aeróbico, fuerza y flexibilidad (intervención), el otro, caminata diaria, (control), con duración de doce semanas. Se tomó pretest y postest con prueba de esfuerzo, peso, índice de masa corporal, perímetro de cintura. Se compararon los resultados. En el grupo intervención, el VO2máx aumentó 27\%, el peso disminuyó $2,2 \%$, el perímetro de cintura bajó el 2\%. En el grupo control no hubo cambios significativos.
\end{abstract}

Palabras clave: consumo máximo de oxigeno (VO2máx), peso corporal, índice de masa corporal, perímetro de cintura, sobrepeso, obesidad, ejercicio físico.

\begin{abstract}
Physical exercise is increasingly becoming important in the treatment of patients with hypertension, overweight and obesity. From the last three decades, it is known that physical exercise has favorable effects in the treatment of hypertension and other risk factors; it is an adjunct in the treatment of cardiovascular disease favors regarding maximal oxygen uptake (VO2max), the aim of the research was to determine the effect of a plan of physical training on $\mathrm{VO}_{2}$ max and weight in elderly hypertensive patients, assigned to IPS CAJASAN Bucaramanga. A quasi-experimental research, forty patients were taken between 60-75 years of age in two equal groups, one with aerobic training, strength and flexibility (intervention), and the other with daily walk (control) one hour, five days a week. In the intervention group the $\mathrm{VO}_{2}$ max increased 27\%, 2.2\% weight decreased, waist circumference decreased in $2 \%$, which is considered significant. In the control group no significant changes in the above parameters.
\end{abstract}

Keywords: maximum oxygen uptake (VO2máx), body weight, body mass index, waist circumference, overweight, obesity, physical exercise.

Fecha de recepción: 12 de diciembre de 2013

Fecha de aprobación: 28 de marzo de 2014

\footnotetext{
* Especialista en Acondicionamiento Físico para la Salud, Medicina del Deporte, Geriatría. Docente asociado programa de Licenciatura en Cultura Física Recreación y Deportes de la Universidad del Atlántico. Barranquilla. Colombia. Grupo de investigación Cedinep. Correo electrónico: jorgeperez@mail.uniatlantico.edu.co

** Magíster en Práctica Pedagógica, Especialista en Educación Especial, Licenciada en Educación Preescolar. Docente Catedrático programa Licenciatura en Cultura Física Recreación y Deportes de la Universidad del Atlántico, Barranquilla, Colombia. Correo electrónico: marirojas8888@hotmail.com
} 


\section{INTRODUCCIÓN}

La enfermedad cardiovascular es la primera causa de muerte en todo el mundo. En Colombia, el panorama es muy similar y Bucaramanga no escapa a esta realidad. La hipertensión arterial (HTA) es uno de los factores de riesgos más importantes para el desarrollo de la enfermedad cardiovascular. Según Varela (2008):

La prevalencia de la hipertensión oscila entre el $10 \%$ y $73 \%$, siendo la primera causa de enfermedad cardiovascular, falla cardiaca y enfermedad cerebro vascular y la segunda causa de enfermedad renal crónica, en el 35\% de los eventos cerebrovasculares y el $49 \%$ de las insuficiencias cardiacas se evidencia esta patología, alrededor de un 50\% de las personas adultas mayores de 60 años sufren la enfermedad (p. 18).

La falta de actividad física está asociada a la aparición de HTA, así como otros factores de riesgo para el desarrollo de la enfermedad cardiovascular como obesidad, dislipidemia, diabetes, enfermedad coronaria, entre otros. La actividad física juega un papel primordial en la prevención y el tratamiento de estas entidades clínicas, por eso la necesidad de realizar un tratamiento adecuado e integral para evitar las complicaciones que estas enfermedades puedan generan. A pesar de los significativos avances en cuanto a farmacoterapia de la enfermedad coronaria y sus factores de riesgo, los medicamentos siguen teniendo efectos secundarios, los costos son elevados. De acuerdo con Rojas (2008), el entrenamiento físico está ampliamente reconocido como parte del manejo terapéutico de esta entidad clínica y los factores de riesgo cardiovascular.

El ejercicio físico adquiere cada vez más importancia en el tratamiento de los pacientes con enfermedades crónicas no trasmisibles (ECNT), hace casi tres décadas se publicó en el American Journal of Cardiology:

Existe un agente que baja el colesterol, los triglicéridos, la presión arterial, el peso, la glicemia, es diurético, controla el apetito, mejora la circulación, ayuda a controlar el estrés, la ansiedad, la depresión, previene la osteoporosis y algunos tipos de cáncer, este agente es el ejercicio físico (Roberts, 1984, pp. 261-262).

Duperly y Anchique (2006) consideran que la evidencia epidemiológica y experimental de los últimos tiempos ha enseñado el claro beneficio del ejercicio físico en el tratamiento y prevención de la enfermedad cardiovascular. No es fácil aislar el efecto del ejercicio de otros factores, pero las investigaciones demuestran efectos característicos de este que permiten prevenir la enfermedad cardiovascular. A pesar del conocimiento al respecto, el sedentarismo en el mundo sigue aumentando. Para Colombia, se aceptan cifras del 70\% al 80\% de la población adulta con niveles insuficientes de actividad física regular.

Otras patologías de alta prevalencia en el mundo y en Colombia son el sobrepeso y la obesidad, íntimamente relacionadas con el sedentarismo. Estas enfermedades están en aumento, según el centro del observatorio en salud para Colombia, Así vamos en salud (2010), fueron responsables de "2.8 millones de muertes en el 2004 y fueron clasificados como el quinto factor de riesgo de muerte a nivel mundial. Siendo el tercero en países de ingresos medios como Colombia, cobrando el $6.7 \%$ del total de muertes".

De acuerdo con esta misma organización, en Colombia la prevalencia de exceso de peso presenta una tendencia ascendente en los últimos cinco años, pasando de $46 \%$ en 2005 a $51,2 \%$ en 2010 . Hubo un aumento mayor en el porcentaje de obesos (2,8 puntos porcentuales). Esta condición es más prevalente en las mujeres $(55,2 \%$ mujeres, $45,6 \%$ hombres) y en el grupo de edad de 50 a 64 años. A nivel departamental, San Andrés y Providencia, Guaviare, Guainía, Vichada y Caquetá presentan las mayores prevalencias, todas superiores al $57 \%$ de su población con exceso de peso.

Cuando se desea disminuir tejido graso a través de la actividad física, se debe usar como combustible la grasa acumulada en el adipocito en forma de triglicéridos (TG), el ejercicio físico aumenta el flujo sanguíneo al tejido adiposo, lo que provoca una movilización de triglicéridos hacia el músculo activo, que van a ser oxidados para producir energía, disminuyendo los depósitos, el flujo sanguíneo en el tejido adiposo de humanos y perros no aumenta de forma localizada, como lo demuestran diferentes autores (Hodgetts et al., 1991; Bulow y Madsen, 1976; Bulow, 1982; Bulow y Madsen, 1978; McArdle, 2000; McArdle, 2002, citados por Santa María, 2013), la mayor lipólisis durante el ejercicio se presenta donde haya mayores depósitos.

La utilización de las grasas como principal fuente energética estará condicionada a la intensidad del ejercicio realizado. Romijn et al. (citados por Santa María, 2013) investigaron la contribución energética de los distin- 
tos sustratos durante tres intensidades diferentes de ejercicio. Se demostró que durante el ejercicio de baja intensidad (25\% V02max) los triglicéridos musculares (TGIM) contribuyen mínimamente a la provisión de energía, los ácidos grasos y la glucosa en plasma parecen ser los sustratos más importantes a esa intensidad, donde las grasas son por lejos el combustible predominante. Si la intensidad del ejercicio es moderada (65\% VO2max), los sustratos en el músculo (TGIM y glucógeno) se tornan más importantes. Los TGIM fueron oxidados a altas tasas, a esta intensidad de ejercicio, mientras que los ácidos grasos plasmáticos fueron utilizados a una tasa levemente más baja, comparado con el ejercicio de baja intensidad. Y por último, a intensidades altas ( $85 \%$ VO2max), el sustrato que predominó fue el glucógeno muscular y se produjo un descenso de los TGIM y de los AG plasmáticos.

El consumo máximo de oxígeno (VO2máx) es el parámetro que se utiliza habitualmente en la valoración del rendimiento cardiocirculatorio. Representa la máxima capacidad de oxígeno transportada y utilizada por el metabolismo celular de los diferentes órganos y sistemas de una persona cuando esta realiza un ejercicio máximo.

La utilización del oxígeno en los procesos oxidativos durante el ejercicio depende de los siguientes procesos fisiológicos: ventilación pulmonar, difusión alveolo capilar, gasto cardíaco y su redistribución, diferencia arteriovenosa de $\mathrm{O} 2 \mathrm{y}$ de las variaciones fisiológicas del 02. El VO2máx es el producto del gasto cardíaco por la diferencia $\mathrm{A}-\mathrm{V}$ de $\mathrm{O} 2$ en el esfuerzo límite: GC x dif A-V O2 = (FC x VS) x dif A-V O2 (Blog de cardiología, 2012).

A mayor VO2, más aptitud física, crece la capacidad aeróbica, mejor posibilidad de realizar las actividades cotidianas, sobre todo en el adulto mayor, donde se pierde masa muscular con los años, esta pérdida es acelerada por la inactividad física, se aumenta tejido graso, produciendo una obesidad sarcopénica, disminuyendo la fuerza. Al tener menos fuerza, disminuye la posibilidad de realizar actividad física, entra en un círculo que favorece la inactividad física y aumenta cada vez más el tejido adiposo.

Salcido et al. (2013) expresan que el consumo máximo de oxígeno ( $\mathrm{VO} 2 \mathrm{max}$ ) es una de las principales variables en el campo de la fisiología del ejercicio y se usa como indicador del entrenamiento cardiovascular de una persona. Asimismo, un incremento del $\mathrm{VO}_{2}$ max es el método más común para demostrar los efectos del entrenamiento, dado que puede incrementarse, como consecuencia de este, o reducirse por falta del mismo. Se ha establecido que la composición corporal es un factor que influye en el resultado del $\mathrm{VO} 2$, teniendo una relación negativa con el porcentaje de grasa corporal, el peso extra que proporciona la grasa corporal puede contribuir a que, en una prueba en la que se tenga que desplazar el cuerpo, la persona con sobrepeso obtenga un menor rendimiento.

Cuando una persona está en sobrepeso u obesidad y disminuye de peso por cualquier método, su VO2max puede aumentar, esto se debe a que su cantidad de fibras musculares es igual, lo que varió fue el porcentaje de grasa, estas fibras musculares que desarrollan una cantidad de energía para desplazar al cuerpo ahora tienen que trasportar un peso más liviano, favoreciendo su desplazamiento. Si una persona está en peso normal y sube de peso, refiere sensación de ahogo al caminar, mientras que si está en sobrepeso y baja al peso normal, la sensación al caminar es de bienestar y disminución de la fatiga que sentía anteriormente; con igual trabajo transporta menos peso.

Cuando se hace actividad física, especialmente aeróbica, se disminuye de peso, aumentando el VO2max, pero también se producen cambios a nivel de las fibras musculares, se produce hipertrofia muscular independientemente de la edad de la persona, con aumento en el número y tamaño de las mitocondrias, que son las encargadas de consumir oxígeno para producir energía (Delmonte, 2008).

Es de suma importancia la realización de planes y programas para el estudio y tratamiento de las poblaciones que padecen HTA, sobrepeso y obesidad, dado los altos costos que ellas generan, los gastos sanitarios en exámenes paraclínicos, como exámenes de laboratorio, radiografía, ecografía, ecocardiograma, prueba de esfuerzo, cateterismo cardiaco, medicamentos, además de los costos por consulta médica, hospitalización, la morbimortalidad asociada a estas patologías, la declinación de la calidad y expectativa de vida. Por ello, también es necesario optimizar el tratamiento.

El siguiente trabajo de investigación tuvo como propósito fundamental determinar el papel del ejercicio físico sobre el consumo máximo de oxígeno (VO2máx) y el peso (masa corporal) en pacientes adultos mayo- 
res hipertensos, adscritos a la institución prestadora de salud (IPS) Cajasan de Bucaramanga. Esto teniendo como hipótesis de trabajo que un plan de entrenamiento físico estructurado aumenta el $\mathrm{VO}_{2}$ max y disminuye el peso corporal.

\section{MATERIALES Y MÉTODO}

Se tomó una muestra por conveniencia de cuarenta pacientes entre los 60 y 75 años de edad, hipertensos, de ambos géneros, pertenecientes al programa de riesgo cardiovascular (RCV) de Cajasan, todos venían realizando una hora de caminata diaria, se dividieron en dos grupos iguales, uno realizó ejercicios físicos de resistencia aeróbica, fuerza, flexibilidad (grupo intervención); la intensidad del ejercicio aeróbico fue valorada con la escala de Borg modificada (manteniéndose entre 4-8 la intensidad del trabajo), el $\mathrm{VO} 2$ de entrenamiento se mantuvo entre el $50 \%$ y $80 \%$ del $\mathrm{VO}_{2 \text { max, calculada por }}$ la frecuencia cardiaca de reserva, a los pacientes se les colocaron pulsómetros, se siguieron los criterios para realizar ejercicio físico del American Collage Sport Medicine (ACSM), la American Heart Association (AHA) y la Organización Mundial de la Salud (OMS).

El plan de ejercicios físicos fue planeado, orientado y evaluado por un profesional de la actividad física, cinco días a la semana, una hora por sesión, durante doce semanas. El otro grupo siguió su rutina de caminata diaria, una hora al día, cinco días a la semana (control), el resto de tratamiento farmacológico y medidas higiénico-dietéticas fueron iguales para ambos grupos, dado que estos pacientes eran atendidos por un equipo multidisciplinario en el programa de RCV.

Se realizaron valoraciones con prueba de esfuerzo máxima, usando el protocolo de Bruce modificado, se tomó el peso, el índice de masa corporal (IMC) y la circunferencia de la cintura a todos los participantes antes de empezar el plan de entrenamiento (pretest) $y$, luego de doce semanas, al finalizar el plan (postest).

El VO2max se calculó de forma indirecta, utilizando la fórmula del Colegio Americano de Medicina Deportiva $(\mathrm{ACSM}), \mathrm{VO} 2 \max =\left(\mathrm{V}^{*} 0,1\right)+\left(\mathrm{G}^{*} \mathrm{~V}^{*} 1,8\right)+35$, donde se relaciona la velocidad de la banda $(\mathrm{V})$ con el porcentaje de inclinación $(G)$. La velocidad de la banda está en $\mathrm{m}^{*} \mathrm{~min}^{-1}$, normalmente se expresa en $\mathrm{Km}^{*} \mathrm{~h}^{-1} \mathrm{o}$
Millas* $\mathrm{h}^{-1}$, por lo que se debe hacer la conversión. El grado de inclinación se coloca en decimales, no en porcentajes, así, por ejemplo, $10 \%$ corresponde a 0,1 . No existe una fórmula que calcule con precisión el $\mathrm{VO}_{2 \max }$ por el Bruce modificado, por lo que se debe usar la propuesta de ACSM, validada a nivel internacional (Hayward, 2008).

Para la tabulación y el procesamiento de la información, se creó una base de datos en Excel 2010, luego se procesó la información en el software estadístico SSPS 20 para Windows. Se utilizaron estadísticas descriptivas que incluyeron medidas de tendencia central, media, mediana, para las variables, edad, $\mathrm{VO}_{2} \max$ y peso, medidas de dispersión como varianza y desviación estándar, para las variables VO2max y peso.

Se pasó por un proceso de discusión, se determinó la diferencia de realizar actividad física espontanea versus un plan estructurado, mirando el impacto en las variables estudiadas.

Se garantizó la protección de la intimidad de las personas participantes de acuerdo con la declaración de Helsinki de 1964 y la disposición de la resolución 008430 del Ministerio de Salud de Colombia de 1993 sobre la firma del consentimiento informado en investigación en seres humanos.

\section{RESULTADOS}

Del análisis estadístico de los resultados se puede observar que la conformación de ambos grupos fue similar en cuanto a distribución por género y el promedio de edad (tabla 1).

Tabla 1. Distribución por género y edad. Ambos grupos.

\begin{tabular}{|c|c|c|c|c|}
\hline & \multicolumn{2}{|c|}{ Grupo control } & \multicolumn{2}{c|}{ Grupo intervención } \\
\hline Género & Cantidad & Porcentaje & Cantidad & Porcentaje \\
\hline Femenino & 17 & $85 \%$ & 16 & $80 \%$ \\
\hline Masculino & 3 & $15 \%$ & 4 & $20 \%$ \\
\hline $\begin{array}{c}\text { Edad } \\
\text { Promedio } \\
\text { (años) }\end{array}$ & 67 & & \\
\hline
\end{tabular}


A continuación, (tabla 2 y gráficos 1 y 2) se muestra una disminución del VO2max en el grupo control, de $-1,05$ $\mathrm{ml}^{*} \mathrm{~kg}^{-1 *} \mathrm{~min}^{-1}$, equivalente al-3,6\%, mientras en el grupo intervención hubo un aumento de 7,05 $\mathrm{ml}^{*} \mathrm{~kg}^{-1 *} \mathrm{~min}^{-1}$, lo que representa un $27 \%$ (es estadísticamente significa- tivo), con un valor $p<0,05$, es decir, se confirma la hipótesis del aumento del VO2max después de hacer el plan de entrenamiento en el grupo intervención. Además, la dispersión fue mayor en el grupo control en relación con el grupo intervención.

Tabla 2. Comparación del consumo máximo de oxígeno (VO2máx) en el pretest y en el postest. Ambos grupos.

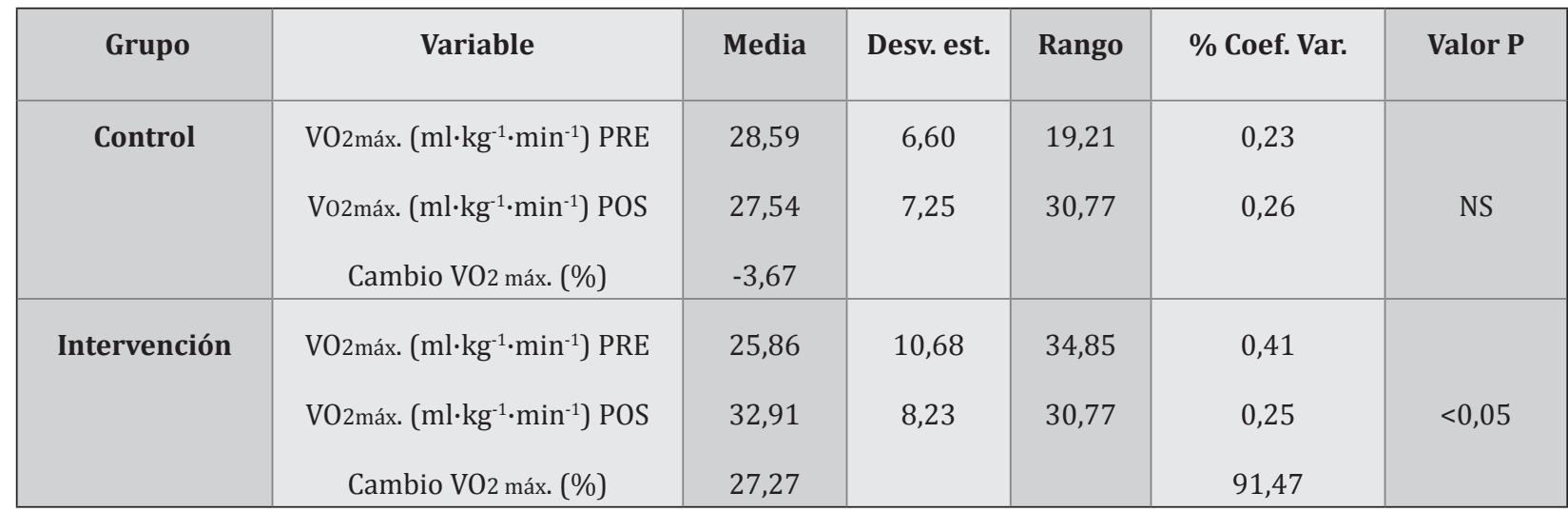

Gráfico 1. Pretest (PRE), postest (POS), NS (diferencia estadísticamente no significativa) valor p<0,05.

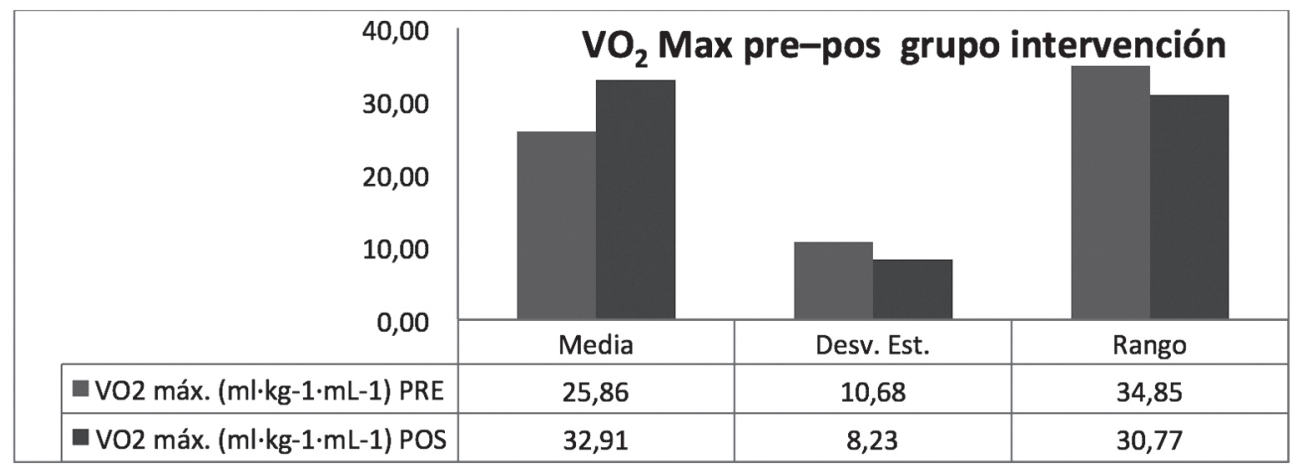

Gráfico 2. VO2 Max pre-pos grupo control

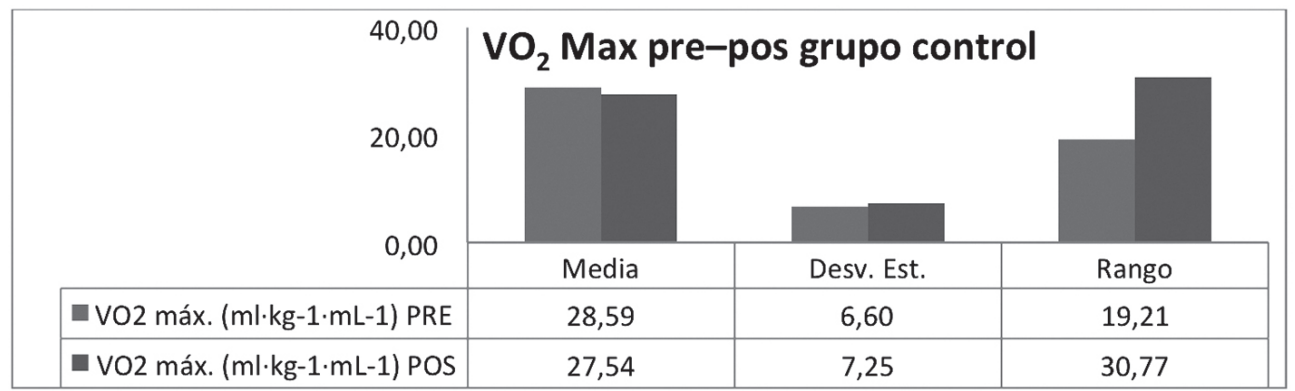

A continuación, se muestra (tabla 3 y gráficos 3 y 4) que en el grupo control se produjo un aumento de $0,35 \mathrm{~kg}$, lo que significa un 0,53\% del peso, mientras en el grupo intervención el peso bajó -1,37 kg, lo que representa un -2,1\% 
Tabla 3. Comparación del peso pretest y del peso postest en ambos grupos.

\begin{tabular}{|c|c|c|c|c|c|c|}
\hline \multirow{2}{*}{ Grupo } & Variable & Media & Desv. est. & Rango & Coef. Var. & Valor P \\
\hline \multirow{2}{*}{ Control } & Peso PRE (Kg) & 65,85 & 11,25 & 39 & 0,17 & NS \\
& Peso POS (Kg) & 66,20 & 12,59 & 39 & 0,19 & 0,16 \\
\hline \multirow{2}{*}{ Intervención } & Cambio del peso (\%) & 0,53 & & & 0,20 \\
& Peso PRE (Kg) & Peso POS en Kg & 65,70 & 13,24 & 46 & $<0,05$ \\
\hline
\end{tabular}

Pretest (PRE), postest (POS), NS (diferencia estadísticamente no significativa) valor p<0,05.

Gráfico 3. Peso pre-pos grupo control

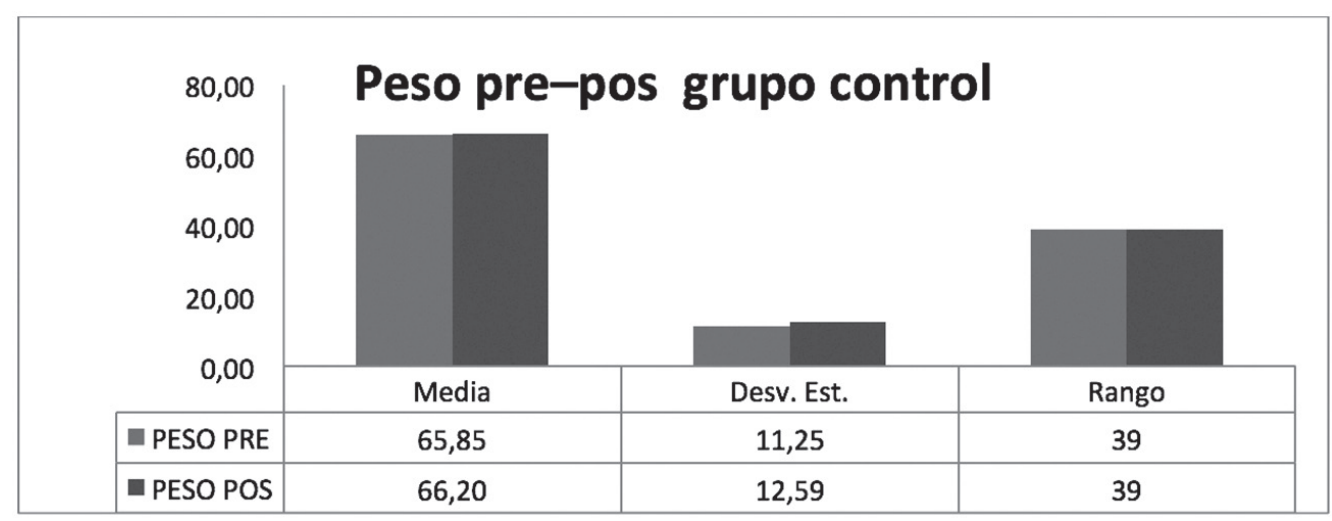

Grafico 4. Peso pre-pos grupo intervención

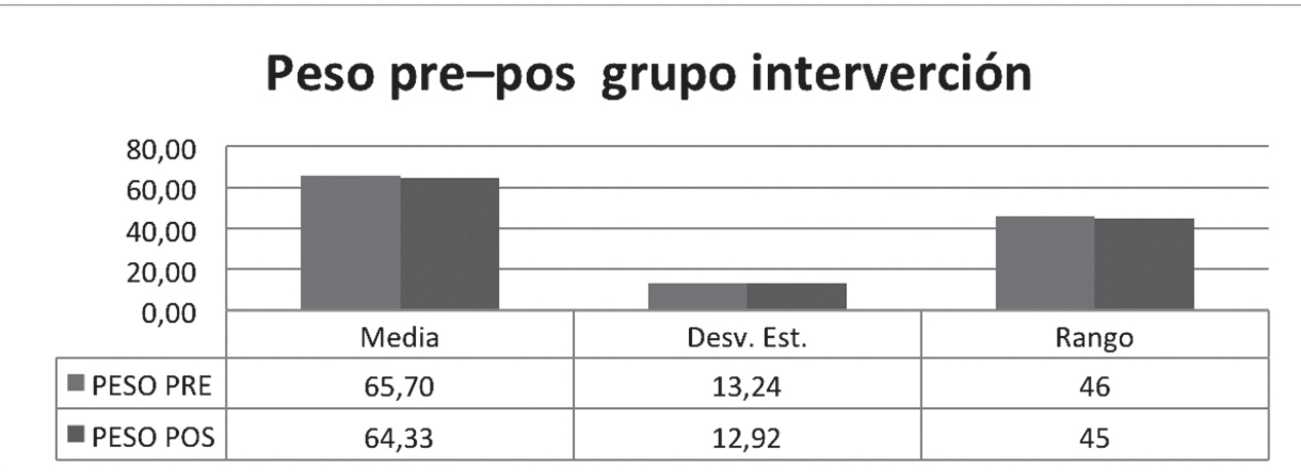

Igualmente, el índice de masa corporal (IMC) aumentó 0,16 Kg*m2 -1, representando 0,57\% en el grupo control, y disminuyó -0,51 Kg*m2 -1, correspondiente al-1,75\% en el intervención (tabla 4). 
SECCIÓN CENTRAL

Tabla 4. Comparación del IMC en el pretest y postest.

\begin{tabular}{|c|c|c|c|c|c|c|}
\hline Grupo & Variable & Media & Desv. Est & Rango & \% Coef. Var. & Valor $\mathbf{P}$ \\
\hline Control & $\begin{array}{l}\text { IMC }(\mathrm{Kg} / \mathrm{m} 2) \text { PRE } \\
\text { IMC (Kg/m2)POS } \\
\text { Cambio IMC (\%) }\end{array}$ & $\begin{array}{r}28,11 \\
28,27 \\
0,57\end{array}$ & $\begin{array}{l}4,80 \\
3,86\end{array}$ & $\begin{array}{l}19,40 \\
16,50\end{array}$ & $\begin{array}{l}0,17 \\
0,14\end{array}$ & NS \\
\hline Intervención & $\begin{array}{l}\text { IMC (Kg/m2)PRE } \\
\text { IMC (Kg/m2)POS } \\
\text { Cambio IMC (\%) }\end{array}$ & $\begin{array}{l}29,11 \\
28,60 \\
-1,75\end{array}$ & $\begin{array}{l}4,57 \\
4,45\end{array}$ & $\begin{array}{l}16,90 \\
16,90\end{array}$ & $\begin{array}{l}0,16 \\
0,16\end{array}$ & $<0,05$ \\
\hline
\end{tabular}

Pretest (PRE) y postest (POS), NS (diferencia estadísticamente no significativa) valor $\mathrm{p}<0,05$.

El perímetro de cintura del grupo control aumento $0,4 \mathrm{~cm}$, lo que significa un 0,44\%, y el del grupo intervención disminuyó-1,85 cm, lo que representa un -2,04\% (tabla 5).

Tabla 5. Comparación del perímetro de cintura en el pretest y postest.

\begin{tabular}{|c|c|c|c|c|c|c|}
\hline Grupo & Variable & Media & Desv. típ. & Rango & \% Coef. Var. & Valor P \\
\hline Control & $\begin{array}{l}\text { Perímetro de cintura }(\mathrm{cm}) \text { PRE } \\
\text { Perímetro de cintura }(\mathrm{cm}) \text { POS } \\
\text { Cambio perímetro }(\%)\end{array}$ & $\begin{array}{l}90,75 \\
91,15 \\
0,44\end{array}$ & $\begin{array}{l}16,47 \\
16,60\end{array}$ & $\begin{array}{l}48,00 \\
48,00\end{array}$ & $\begin{array}{l}0,18 \\
0,18\end{array}$ & NS \\
\hline Intervención & $\begin{array}{l}\text { Perímetro de cintura(cm) PRE } \\
\text { Perímetro de cintura(cm) POS } \\
\text { Cambio perímetro }(\%)\end{array}$ & $\begin{array}{r}90,4 \\
88,55 \\
-2,04\end{array}$ & $\begin{array}{l}14,95 \\
14,45\end{array}$ & $\begin{array}{l}49,00 \\
48,00\end{array}$ & $\begin{array}{l}0,17 \\
0,16\end{array}$ & $<0,05$ \\
\hline
\end{tabular}

Pretest (PRE) y postest (POS). NS (diferencia estadísticamente no significativa) valor $\mathrm{p}<0,05$. 


\section{ANÁLISIS Y DISCUSIÓN}

En ambos grupos, la distribución por género y edad fue similar, lo que hace pertinente la comparación de los resultados, la mejoría del VO2máx del $27 \%$ en el grupo intervención indica aumento de la capacidad física, por ende, mayor posibilidad para realizar sus labores diarias, más capacidad y autonomía, sobre todo en este rango de edad, donde se va perdiendo la aptitud física y la posibilidad para realizar las actividades, los instrumentales y las actividades avanzadas de la vida diaria. De igual manera, nos indica un mejor funcionamiento cardiovascular.

De Miguel et al. (2011) realizaron en España una investigación con 105 trabajadores en edades entre los 23 y 57 años de edad de una empresa, se trabajó resistencia, fuerza y flexibilidad de 3 a 4 veces por semana, se hizo control del VO2max a los 6 meses y al año, encontrando que había mejorado de $41,1 \mathrm{ml} \cdot \mathrm{kg}^{-1} \cdot \mathrm{min}^{-1}$ inicial a $44 \mathrm{ml} \cdot \mathrm{kg}^{-1} \cdot \mathrm{min}^{-1}$ a los 6 meses $(7,05 \%)$ y de $47,1 \mathrm{ml} \cdot \mathrm{kg}^{-1} \cdot \mathrm{min}^{-1}$ al año $(14,6 \%)$. Pollock (citado por De Miguel et al., 2011) considera que un incremento entre $15 \%$ y $20 \%$ es más normal para una persona media que fuera sedentaria antes del entrenamiento y que se entrena al $75 \%$ de su frecuencia cardiaca máxima tres veces por semana, $30 \mathrm{~min}$ al día, durante 6 meses.

Otro estudio realizado por García et al. (2010) en Murcia, España, tuvo como propósito determinar los efectos de un entrenamiento mixto de 6 semanas de duración sobre la condición física de mujeres jóvenes con sobrepeso. La muestra estuvo compuesta por 20 mujeres de 19 años en promedio, el entrenamiento se llevó a cabo durante 6 semanas, ejecutándolo 3 veces por semana con una duración de 40 a 50 minutos por sesión. Antes y después del programa, se evaluó consumo máximo de oxígeno (VO2máx), se observó un incremento del 7\%, como vemos, la ganancia del $\mathrm{VO}_{2 m a ́ x}$ fue relativamente poca, pero el tiempo también fue muy reducido para obtener resultados más significativos.

A los pacientes del grupo intervención del presente estudio no solo se les realizó trabajo aeróbico, sino también de fuerza, el mejor fitness muscular (la fuerza y la resistencia muscular) proporcionan considerables beneficios a las personas mayores. El entrenamiento contra resistencia puede permitir a los ancianos realizar con mayor facilidad las actividades de la vida diaria y contrarrestar la debilidad y fragilidad muscular propia de la edad, según el ACSM (2006). Es muy importante que el participante mantenga un mínimo de fitness muscular si quiere seguir valiéndose por sí mismo.
De acuerdo con Ramos et al. (2010), ha sido ampliamente comprobado que el V02máx disminuye con la edad, debido a un deterioro en un $25 \%$ del gasto cardíaco máximo y al descenso de la utilización periférica del 02 , dada la reducción en una cuarta parte de la diferencia arteriovenosa de 02 , así como valores menores de frecuencia cardiaca máxima, de volumen máximo de eyección y de los parámetros de la función pulmonar.

Es importante realizar actividad física que sea benéfica para la salud, sin causar lesiones, en los adultos mayores. Una intensidad muy alta podría producir lesiones en el aparato locomotor, mayor probabilidad de osteoartrosis, por sobreuso de las articulaciones y mayor impacto en estas estructuras, lo que generaría mayor desgaste. Igualmente, su aparato cardiovascular no debe sobrecargarse para evitar problemas de hipertensión, desencadenamiento de un episodio isquémico coronario, desordenes metabólicos, que en esta edad y con las comorbilidades preexistente es muy riesgoso.

La pérdida de peso en el grupo intervención fue de $2,1 \%$, en el grupo control hubo una ganancia de $0,53 \%$. Se debe aclarar que no todos necesitaban bajar de peso, dado que algunos estaban dentro del rango considerado normal. Según la Organización Mundial de la Salud (OMS 2012), el criterio de normalidad del índice de masa corporal (IMC) para pacientes de la tercera edad es de 20-27 y sobrepeso de 28-30.

De acuerdo con este criterio, encontramos que en el grupo intervención el 30\% tiene peso normal, el 35\%, sobrepeso y el $35 \%$, obesidad, en el grupo control, el $45 \%$ es normal, el $35 \%$ presenta sobrepeso y el $20 \%$, obesidad.

El IMC aumentó 0,57\% en el grupo control mientras en el grupo intervención bajó 1,75\%, también se puede ver que el perímetro de cintura en el grupo intervención disminuyó $2,0 \%$, y en el grupo control aumentó $0,44 \%$, lo que se correlaciona con la pérdida de peso.

Pratley (citado por Torres et al., 2010) aplicó un programa de 9 meses de duración en adultos obesos con base en la carrera y el ciclismo, donde la carga evolucionó de 3 a 4 sesiones semanales, la intensidad ascendió del 50 al 80\% y la duración de 30-45 minutos a 45-60 minutos, encontrando reducción del $2 \%$ en el perímetro de la cintura.

Villarreal et al. (2013) realizaron una investigación en Nuevo León, México, con el objetivo de demostrar los efectos de la intervención psicoeducativa en el manejo 
de la obesidad, en una muestra de 60 mujeres entre los 20 y los 60 años de edad con IMC superior a 27, se dividieron en dos grupos, uno control y otro intervención, el primero con tratamiento de dieta, el segundo con dieta y modificación de hábitos de vida, especialmente aumento de la actividad física, las conclusiones del estudio fueron que hubo cambios significativos en el grupo intervención, pérdida de peso de 2,6 kg, el IMC disminuyó 1,2 y disminución de la circunferencia de la cintura de 2,3 cm al haber aumentado el número y la duración de las actividades físicas, más que con la exclusiva modificación de los hábitos dietéticos.

Habitualmente, la obesidad se trata modificando la dieta, pero no es esta la única variable relacionada con la pérdida de peso. La actividad física contribuye a la pérdida de peso al ser un medio que proporciona un déficit energético, las evidencias científicas apoyan que la combinación de modificaciones en la dieta y ejercicio físico es el método más efectivo para conseguir abordar la pérdida de peso, siendo la realización de ejercicio duradera uno de los mejores predictores de un mantenimiento prolongado de la pérdida de peso. Según Estévez, Tercedor y Delgado (2012):

La recomendación general de $150 \mathrm{~min} \cdot \mathrm{sem}^{-1} \mathrm{de}$ actividad física aeróbica de intensidad moderada también es aplicable para las personas con sobrepeso u obesidad, porque disminuirá el riesgo de padecer enfermedades cardiovasculares. Esta recomendación general constituye también la mínima específica para prevenir ganancias de peso no saludables, para lo cual se establece un margen que va desde los 150 hasta los $250 \mathrm{Kcal} \cdot \mathrm{sem}^{-1}$. Algunos estudios centran su atención en el consumo energético provocado por la realización regular de actividad física más que en las características de la propia actividad física. Existe consenso en indicar que un gasto energético de $1000 \mathrm{Kcal} \cdot \mathrm{sem}^{-1}$ parece ser insuficiente para prevenir ganancias de peso. Por ello, las recomendaciones emitidas sugieren alcanzar un gasto energético mínimo de 1500-2000 Kcal·sem-1 para mantener el peso (p. 236).

Si bien los cambios en la composición corporal son consecuencia de un proceso multifactorial y se producen a lo largo del proceso de envejecimiento incluso en personas sanas, existen evidencias de que el estilo de vida juega un papel de especial relevancia sobre la masa grasa, muscular y ósea. Concretamente, la OMS aboga por la nutrición y la actividad física como facto- res de gran influencia sobre la composición corporal de las personas mayores.

Burgos (2006) considera que el incremento del peso y la masa grasa durante la primera etapa del envejecimiento puede estar originado por el descenso del gasto de energía total derivado de una disminución de la actividad física y del metabolismo basal en presencia de una ingesta calórica estable o aumentada. Por el contrario, existen multitud de factores que durante la senectud contribuyen a un déficit en la ingesta y que suelen conllevar un aumento del ritmo de pérdida de masa corporal, muscular y ósea en este grupo de población. Independientemente de la causa, la pérdida de peso corporal se asocia con la aparición y desarrollo de la sarcopenia y la osteoporosis por una aceleración de la pérdida de masa muscular y masa ósea. Con el aumento del nivel de sedentarismo que acontece durante el envejecimiento, se produce un aumento de la masa grasa y descenso de la masa muscular, que al mismo tiempo favorece que la actividad física se vuelva cada vez más escasa, al haber menos masa muscular hay pérdida de fuerza y menos capacidad para realizar actividad física, esta inactividad genera más perdida muscular, lo que genera un círculo vicioso.

Gómez et al. (2012) plantean que hay suficientes estudios que demuestran que tanto la actividad física, medida preferiblemente a través de acelerómetros, como programas específicos de entrenamiento son capaces de revertir (al menos parcialmente) los cambios de la composición corporal en personas mayores inicialmente sedentarias, esto indica que un estilo de vida activo es capaz de preservar la masa muscular, masa grasa y masa ósea en unos niveles saludables. También se ha demostrado que las personas físicamente activas durante su vida tienen menor riesgo de padecer enfermedades relacionadas con la composición corporal que aquellas personas con un estilo de vida sedentario.

Un dato interesante es que la pérdida de peso que se consigue por la práctica de actividad física tiende a colaborar de manera eficaz en la movilización de la grasa acumulada a nivel abdominal. La actividad física tiende, tanto en hombres como en mujeres, a proporcionar efectos beneficiosos sobre la circunferencia de cintura, reduciéndola significativamente en comparación con sujetos sedentarios. 
En cualquier caso, en personas adultas, el mayor beneficio para la pérdida de grasa se obtiene cuando ambas estrategias, dieta y ejercicio, se asocian. La actividad física contribuye, además, a mantener durante más tiempo la pérdida de peso conseguida, aumentando la oxidación de las grasas, preferentemente de la región central del cuerpo. Prescribir programas de ejercicio en esta dirección constituye un enfoque fundamental para conseguir éxito en la población obesa.

Martínez (2000) considera que el tratamiento de la HTA debe ser integral, y no solo se debe tratar la HTA, sino los otros factores de riesgo asociados. Se postula que el agente antihipertensivo ideal sería aquel que sea bien tolerado, efectivo y seguro en la reducción de la presión arterial, conveniente en la posología, de bajo costo, al alcance de todos, sencillo en su aplicación, sin efectos secundarios indeseables y, de ser posible, que contribuya a revertir las disfunciones creadas en el hipertenso. Todas esas condiciones las cumple el ejercicio físico regular, que, en efecto, reduce eficientemente la presión arterial. Este hecho resulta además bastante atractivo en el manejo de la hipertensión si se tiene en cuenta que, a diferencia de otros tratamientos, en lugar de producir efectos secundarios indeseables, tiene una serie de consecuencias, todas ellas muy favorables para la salud física y mental de los individuos.

Existen hoy argumentos científicos para afirmar la existencia de una relación inversa entre nivel de actividad física y factores de riesgo cardiovascular. Esta relación es más consistente cuando se trata de actividad física de vigorosa intensidad que cuando se trata de actividad física de menor intensidad. Según el ACSM (2006), cualquier nivel de actividad física es mejor que ninguna, pues, como mínimo, ayuda a regular el equilibrio entre la ingesta y el gasto energético.

Cuenca et al. (2011) consideran que la práctica de actividad física, con un componente de intensidad moderada-vigorosa, debe estar presente ya desde la infancia o la adolescencia. Esta es la clave para mantener un estilo de vida saludable; si se aumenta la actividad física, se podrían evitar el sobrepeso y la obesidad y disminuir de esta forma la aparición o ayudar en el control de otras enfermedades como la hipertensión, la diabetes, el aumento del colesterol, los triglicéridos y la aparición de enfermedad coronaria.
Otro aspecto fundamental en el tratamiento adecuado de los pacientes con factores de riesgo cardiovascular es capacitar al personal de salud sobre estos temas; para Colbert y Jangi (2013), la formación que poseen los médicos sobre estos temas es escasa, se deben hacer modificaciones curriculares para el estudio e investigación de esos temas. Se aprende mucho sobre el tratamiento de las enfermedades agudas y crónicas, sus complicaciones, pero se debe enfatizar en la prevención a través del ejercicio físico y la dieta. Sobre todo cuando los pacientes son jóvenes, las posibilidades de cambios conductuales son mayores y los daños en órgano blanco son menores. Algunas escuelas de medicina en Estados Unidos lo están haciendo con gran éxito, incluyendo trabajo multidisciplinario y educación a la comunidad.

\section{CONCLUSIONES Y RECOMENDACIONES}

En el presente estudio, se comprobó el efecto benéfico del ejercicio físico estructurado, orientado por un profesional de la actividad física, en comparación con el ejercicio autodirigido, sobre el VO2máx y el peso corporal en pacientes adultos mayores hipertensos afiliados a la IPS Cajasan Bucaramanga. Es de vital importancia que un profesional de la actividad física sea quien planifique, oriente, estructure y evalúe estos entrenamientos, es él quien puede medir las cargas del entrenamiento, la duración, intensidad, la frecuencia, el tipo de ejercicio y su progresión, con criterios científicotécnicos sobre cómo se deben trabajar los volúmenes, en especial en poblaciones como la tercera edad. El ejercicio físico es una medicina que se debe saber prescribir igual que cualquier otro medicamento.

El ejercicio físico estructurado diario aumentó el VO2máx en un $27 \%$, disminuyó el peso corporal en 2,1 \% y el perímetro de cintura en $2 \%$. Esto es importante dado que la obesidad abdominal es la que está directamente relacionada con patologías cardiovasculares.

Es importante mejorar el VO2máx en los ancianos, porque a mayor consumo de oxígeno, mayor capacidad para realizar sus labores diarias, más autonomía; en esta edad son muy frecuentes las caídas, un anciano frágil está más propenso a caída, fractura e incluso la muerte; pero el beneficio no solo es sobre el aparato locomotor, sino cardiovascular. Los programas de ejer- 
cicio físico deben estar constituidos de trabajo aeróbico, fuerza, equilibrio y flexibilidad. Las cargas de entrenamiento se deben ir aumentando paulatinamente, no se deben aumentar de manera abrupta que ponga en riesgo la salud de los participantes. Es nece-

\section{REFERENCIAS BIBLIOGRÁFICAS}

American College of Sport Medicine. (2006a). Manual ACSM para la valoración y prescripción del ejercicio. Barcelona: Paidotribo.

American College of Sport Medicine. (2006b). Manual de consulta para el control y la prescripción del ejercicio. Barcelona: Paidotribo.

American Heart Association (AHA) (s.f.). www.heart.org/ heartory.

Así vamos en salud. (s. f.). Prevalencia de la obesidad, sobrepeso y exceso de peso- goediferenciado. Recuperado de http://www.asivamosensalud.org/ inidicadores/estado-de-salud/grafica.ver/61.

Burgos, R. (2006). Sarcopenia en ancianos. Endocrinología y Nutrición, 52, (5).

Colbert, J. y Jangi, S. (2013). Training Physicians to Manage Obesity-Back to the Drawing Board. Engl J Med, 369(15), 10.

Cuenca, M., Ruiz, J., Ortega, F. y Castillo, M. (2012). Actividad física en niños y adolescentes. Recuperado de http// www.Revistasdigitales.csd.gob.es/index. php/ICD/article/viiew/618.

De Miguel, J., Schweiger, I., De las Mozas, O. y Hernández, J. (2011). Efecto del ejercicio físico en la productividad laboral y el bienestar. Revista de Psicología del Deporte, 20, (2), 589-604.

Delmonte, H. (2008). Prescripción del ejercicio físico en el adulto sano, medicina, ejercicio y deporte. Buenos Aires: Fundación Favaloro.

Duperly, J. y Anchique, C. (2006). Actividad física y prevención cardiovascular. Bogotá: Asociación colombiana de Medicina Interna.

Estévez, F., Tercedor, P. y Delgado, M. (2012). Recomendaciones de actividad física para adultos sanos. Journal of Sport and Health Research, 4, (3), 233-244.

García, M., Calahorro, F., Torres, A. y Lara, A. (2010). Efectos de un programa de entrenamiento mixto sobre la condición física en mujeres jóvenes con sobrepeso. Cuadernos de Psicología del Deporte Dirección General de Deportes, 10, 11-16. sario impulsar la implementación de programas de actividad física como coadyuvante en el tratamiento de los pacientes que pertenecen a los programas de promoción y prevención de las IPS y de las EPS.

Gómez, A., Rodríguez, G., Vila, S., Casajús, J. y Ara, I. (2012). Envejecimiento y composición corporal: la obesidad sarcopénica en España. Nutr. Hosp., 27, (1).

Hayward, V. (2008). Evaluación de la aptitud física y prescripción del ejercicio. Buenos Aires: Panamericana.

Martínez, E. (2000). Ejercicio físico y salud. IATREIA/ Revista de la Facultad de Medicina de la Facultad de Medicina Ude A, 13, (4).

Ministerio de Salud Pública de Colombia. (1993). Resolución 008430, por la cual se reglamenta la investigación en seres humanos en el país.

Moraga, C. (2008). Prescripción del ejercicio físico en pacientes con hipertensión arterial. Rev. Cost de Cardio, 10, (1-2), 123-129.

Organización Mundial de la Salud (OMS). (2010). Recomendaciones mundiales sobre Actividad Física para la Salud. Recuperado de hqlibdoc.who.int/ publications/2010/9789243599977_spa.pdf.

Organización Mundial de la Salud (2012). Obesidad y sobrepeso. Recuperado de http://www.who.int/ mediacentre/factsheets/fs311/es/.

Ramos, C., Santos, A., Ochoa, M., Peña, N., Ramos, R. y Ramos, R. (2010). Comportamiento fisiológico cardiorrespiratorio en el adulto mayor durante el ejercicio físico. Recuperado de http://www.cocmed. sld.cu/no143/no143rev01.htm.

Roberts, C. (1984). Editor in chief. American Journal of Cardiology, 53, 261-262.

Salcido S. et al. (2013). Comparación del consumo máximo de oxigeno, ajustado a la masa muscular, entre jóvenes con sobrepeso y peso normal, con moderado nivel de actividad física. Recuperado de EFdeportes.com.

Santa María, M. (2013). Metabolismo de los lípidos durante el ejercicio físico. Recuperado de http://g-se.com/ es/org/cardioactivo/articulos/metabolismo-de-loslipidos-durante-el-ejercicio-fisico-1608.

Sin autor. (2012). El consumo de oxígeno y el consumo miocárdico de oxígeno. Recuperado de http// www. elbogde cardiología/ cardiología-deporte-mioc 
Torres, G., García, M., Villaverde, C. y Garatachea, N. (2010). Papel del ejercicio físico en la prevención y tratamiento de la obesidad en adultos. Retos. Nuevas tendencias en Educación Física, Deporte y Recreación, Revista de la Federación Española de Asociaciones de Docentes de Educación Física (FEADEF) 18, 47-51.

Varela, E. (2008). Aspectos epidemiológicos de la hipertensión arterial. Rev. Colom cardio, guías colombianas para el diagnóstico y tratamiento de la HTA. Boletín 1.
Villarreal, T., Garza, T., Gutiérrez, R., Méndez, E. y Ramírez, J. (2013). Intervención psicoeducativa en el tratamiento de la obesidad en un centro de salud de Nuevo León, México. Recuperado de www.journals.unam.mx/index. php/atencion_familiar/.../30765.

$18^{\circ}$ Asamblea Médica Mundial. (1964). Declaración de Helsinki de la asociación médica mundial, principios éticos, para las investigaciones médicas en seres humanos. 